\title{
Experiences of Designing and Deploying Intelligent Sensor Nodes to Monitor Hand-Arm Vibrations in the Field
}

\author{
Christos Efstratiou \\ efstrati@comp.lancs.ac.uk \\ Joe Finney \\ joe@comp.lancs.ac.uk
}

\author{
Nigel Davies \\ nigel@comp.lancs.ac.uk \\ Rob Hooper \\ r.p.hooper@lancaster. \\ ac.uk
}

\author{
Gerd Kortuem \\ kortuem@comp.lancs.ac.uk \\ Mark Lowton \\ m.lowton@lancaster.ac.uk
}

Computing Department, InfoLab21, Lancaster University

Lancaster LA1 4WA, United Kingdom

\begin{abstract}
The NEMO project is exploring the use of mobile sensor nodes to augment physical work artefacts in order to ensure compliance with health and safety regulations. In this paper we present our experiences of designing and deploying the NEMO Hand Arm Vibration (HAV) monitoring system. Long term exposure to hand arm vibration can lead to serious health conditions and the NEMO HAV monitoring system offers an integrated architecture for capturing HAV exposure data in the field, providing feedback about exposure levels both in the field and as input to a back-end database. Our design allows health and safety regulations specified at the enterprise level to be embedded within the wireless sensor nodes allowing them to operate without any infrastructural support. The system has been the subject of a two week field trial that took place with the collaboration of a British construction and maintenance company. During the field trial, the NEMO HAV system was deployed to a road maintenance patching gang and data was collected on HAV exposure caused by hydraulic drills. The paper reports on the results of the field trial and the lessons learned through the real deployment of the system.
\end{abstract}

\section{Categories and Subject Descriptors}

C.3 [Special-Purpose and Application-Based Systems]: Real-time and embedded systems; J.7 [Computers in Other Systems]: health and safety in industry; C.2.1 [Network Architecture and Design]: Wireless communication

\section{General Terms}

Experimentation, Design

\section{Keywords}

Health and safety, ad-hoc networks, embedded systems, mobile systems, hand-arm vibration, deployment

Permission to make digital or hard copies of all or part of this work for personal or classroom use is granted without fee provided that copies are not made or distributed for profit or commercial advantage and that copies bear this notice and the full citation on the first page. To copy otherwise, to republish, to post on servers or to redistribute to lists, requires prior specific permission and/or a fee.

MobiSys'07, June 11-13, 2007, San Juan, Puerto Rico, USA.

Copyright 2007 ACM 978-1-59593-614-1/07/0006 ...\$5.00.

\section{INTRODUCTION}

Many physical work environments such as construction sites, factories and storage depots, are extremely hazardous and each year numerous people are injured in work-related accidents. To ensure workers' health and safety legal authorities such as national governments or industrial bodies, define health and safety $(\mathrm{H} \& \mathrm{~S})$ regulations that industrial organisations must comply with. These regulations define goals and general policies that - when followed - promote health and safety in the workplace. Policies may take the form of tasks that need to be performed or dictate limitations in certain aspects of a job. However, only rarely do policies prescribe the methods or techniques that companies need to employ in order to achieve the outlined health and safety goals. This leaves it up to industry to find effective and efficient ways to comply with health and safety regulations. Companies need to be able to establish that they comply with regulations, in front of governing bodies, insurance companies or legal authorities. While this can be done in several ways, it commonly involves data collection and record keeping, a process that is time consuming and costly.

Currently, most health and safety systems rely on human information gathering and recording in the field, with decisions being taken by workers, supervisors or back office staff processing the data off-line. In the NEMO project we are exploring an alternative vision in which H\&S support is provided by physical work artefacts, such as tools, augmented with cooperating mobile nodes featuring both sensors and actuators and communicating over ad-hoc wireless networks. These mobile systems would be able to observe the working activities taking place, evaluate compliance with health and safety regulations and assist or actively enforce compliance with these regulations. This vision creates a new field of work in the area of mobile computing support for health and safety and in [5] we have outlined some of the challenges associated with this domain.

In this paper we focus on our experiences of designing, implementing and evaluating a prototype Hand Arm Vibration (HAV) monitoring system based on the NEMO vision of augmented work artefacts. Long term exposure to hand arm vibration can lead to serious health conditions such as "vibration white finger" (VWF), and existing health and safety regulations specify limits on workers' exposure to HAV when 
operating heavy vibrating machinery such as hydraulic drills and breakers. Our experimental system comprises a collection of tools, augmented with wireless sensor nodes, personal user devices and mobile computers that collaborate in an ad-hoc manner in order to collect HAV exposure information. This information is available in real-time to workers in the field and subsequently to management via a back-end database. Our design allows health and safety regulations specified at the enterprise level to be embedded within the wireless sensor nodes, allowing them to operate without any infrastructural support. This is crucial for workers who often have to operate in areas with limited or no wireless communication infrastructure. The system was evaluated through a two week field trial that took place with the collaboration of a major UK construction and maintenance company.

\section{DOMAIN ANALYSIS}

\subsection{The Hand Arm Vibration Problem}

Long-term exposure to hand arm vibration can lead to serious health conditions such as "vibration white finger" (VWF) and in extreme cases to life-long disability. VWF is triggered by excessive use of vibrating machinery such as hydraulic drills and breakers, and causes the fingers to become numb and begin turning white. As the disease progresses it can become irreversible; the person suffers increasingly frequent painful attacks at any time and may even lose their fingers (typically this only happens in extreme cases, for example, when people are working with vibrating machinery in very cold conditions, as is the case in the forestry industry among people working with chainsaws).

Extensive health and safety regulations exist to limit workers' exposure to HAV. For example, in 2005 the UK Parliament introduced the Control of Vibration at Work Regulations [10]. These regulations set limits for daily exposure to vibration, define methods for their calculation and formulate guidelines for monitoring an employee's exposure to vibration. The guidelines place a responsibility on employers to assess each individual employee's exposure risk and to consider the specific working conditions of each employee. When it is not possible to eliminate a worker's exposure to vibration then suitable health surveillance must be put in place and immediate action be taken if specific vibration limits are exceeded.

The damage caused by exposure to vibrations is a combination of both the frequency of the vibrating tool and the duration of the exposure. Using a tool that vibrates at a low frequency for a long time can be as damaging as using a heavily vibrating tool for a short time. Thus regulations specify two figures for exposure duration, a limit to the overall daily trigger time (Daily exposure limit), and a limit on short-term exposure to very high levels of vibration (Daily exposure action). These limits are defined in terms of the average daily exposure dose $\mathrm{A}(8)$ :

- Daily exposure limit value $=A(8)$ of $2.5 \mathrm{~m} / \mathrm{s}^{2}$

- Daily exposure action value $=A(8)$ of $5.0 \mathrm{~m} / \mathrm{s}^{2}$

$A(8)$ is defined as $a_{h v} \sqrt{T / 8}$ where

$a_{h v}=$ actual vibration acceleration rate expressed in $\mathrm{m} / \mathrm{s}^{2}$

$T=$ actual exposure duration expressed in hours
In turn, $a_{h v}$ is composed of the root-mean-square acceleration magnitudes in three orthogonal directions, $x, y$ and $z$, at the vibrating surface in contact with the hand. Where daily vibration exposure $A(8)$ is above $2.5 \mathrm{~m} / \mathrm{s}^{2}$ but below $5.0 \mathrm{~m} / \mathrm{s}^{2}$, the following steps should be taken: Inform workers of risk, carry out regular health surveillance of worker, record assessment. Where daily vibration exposure exceeds $5.0 \mathrm{~m} / \mathrm{s}^{2}$, the following actions must be taken: Limit usage immediately, rotate workers, introduce other working methods to reduce HAV. Since in practice $a_{h v}$ is not known for a specific piece of work, equipment manufacturers publish estimated $a_{h v}$ values for each individual tool, which can be used to estimate an operator's exposure.

There is a distinct lack of automated solutions for assisting with HAV H\&S rule compliance. For example, vibration exposure data is typically manually recorded by operatives on paper sheets, which are then entered by hand into a health and safety information system. Moreover, key data such as trigger time must be estimated by operatives, most often hours after work has been completed. This of course raises serious concerns with respect to completeness, accuracy and consistency of captured data. The current practice can be improved using mobile data entry solutions based on handheld wireless computers. Yet, while mobile solutions reduce the need for paper forms they still suffer from the fact that they rely on human information gathering and recording in the field. In addition, current solutions are tailored for off-line processing of data in the back office, ignoring the potential benefits for real-time information in the field.

\subsection{Requirements Capture}

In close collaboration with a major UK company that carries out road maintenance and construction operations, we set out to design, implement and evaluate a Hand Arm Vibration (HAV) monitoring system, based on our approach of augmenting work artefacts. Our team consisted of computer scientists, psychologists, ethnographers and organisational management experts. Together, we engaged with the company on various levels, ranging from high-level management to workers in the filed, to investigate current work practices, identify technical and usability requirements, and anticipate deployment challenges. The following gives an outline of our approach and introduces the requirements that we gathered.

\subsubsection{Methodology}

To inform the design and elicit requirements we followed a two-pronged approach. On the one hand, we undertook extensive work place studies and interviews to understand current work practices. On the other hand, we generated design sketches to elicit concrete feedback.

In total we conducted over twenty formal interviews with managers and employees and 16 full days of work observation. Field observation days also involved informal discussions with upwards of 25 operatives going about their daily work, with the fieldwork being conducted across three different regional sections of the company, each of which with its own safety culture and traditions. An important result with respect to risks from vibration and noise exposure ("invisible risks") was that it emerged that these were appropriately assessed and their mitigation documented at an organisational level, yet were often underestimated or viewed as insignificant by operatives due to the apparently loose linkage between the risk and its outcomes. For exam- 


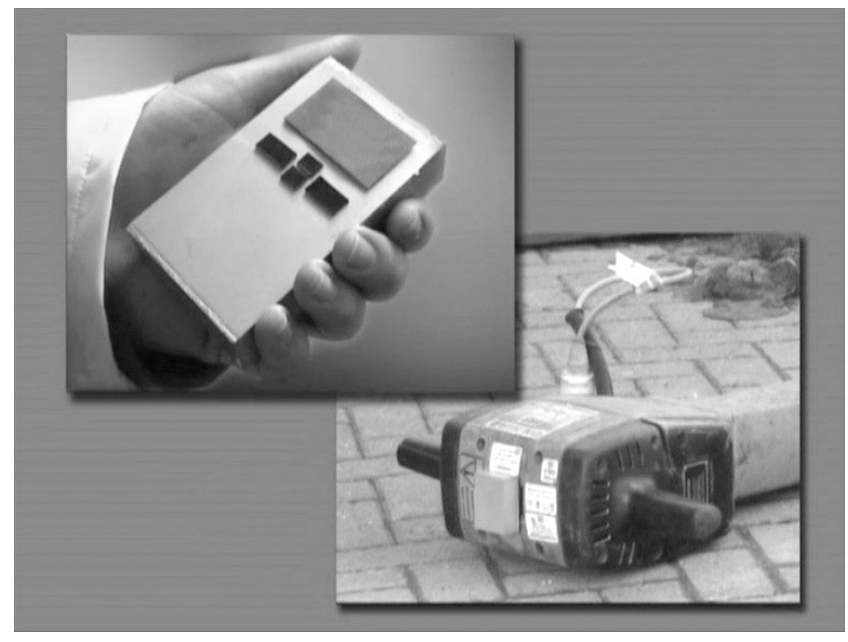

Figure 1: Screen shot of the video sketch used for requirements capturing. The figure shows a mockup of a hand-held user device and a heavy duty jackhammer augmented with a mockup sensor node.

ple, asking operatives about the risks of hand arm vibration was regularly met with a variation on "well, I've been doing this job for twenty years and I haven't had any problems with it". Whilst everyone seemed to know someone who had suffered from vibration white finger through excessive exposure to vibration, this was insufficient to make the risk appear "real" in relation to their own conduct.

We produced design sketches in the form of videos that showed technology mockups operated by members of the design team. These videos were shown to management and operatives alike to force a discussion about concrete design choices (see Figure 1). We found video sketches to be an extremely helpful means to bridge the gap between researchers with a technology vision and practitioners concerned with getting their job done. The design that emerged over time consisted of two different types of devices, a wireless sensor node that can be attached to various pieces of equipment to measure vibrations, and a user device with a display for use by operatives. The user device builds on the concept of a dosimeter for radiation detection and extends it to the realm of vibration exposure. Using video sketches as well as hardware mockups we were able to discuss and settle issues related to form factor, user interaction and device placement, among other things.

\subsubsection{Requirements}

The requirements that we collected included usability requirements, data requirements and operational requirements. Some of the key requirements, are listed below; the identifiers $\mathbf{U}, \mathbf{D}$ and $\mathbf{O}$ indicate the type of requirement, usability, data and operational respectively:

U1 The system must not require users to perform extensive setup or configuration tasks in the field. Time pressure, protective clothing (gloves), and users' unwillingness to engage with technology put severe constraints on the user interface.

U2 Users must be able to interact with the system with minimal training. The rationale for this is the skill set of the targeted user population and the frequent use of temporary workers.

U3 User devices must be generic and reusable, so that a broken unit can be replaced with a new one. This requires data to be replicated seamlessly across device and infrastructure.

D1 The system must generate accurate, personalized vibration exposure records for each equipment operator. This is crucial to enable companies to demonstrate compliance.

D2 Equipment operators must have access to their personal vibration record while operating equipment, i.e. in the field. Compliance issues (e.g., reaching daily exposure limits) must be indicated immediately.

D3 The system must be able to generate company-wide compliance reports.

O1 The system must be able to operate without connection between devices in the field and backend infrastructure. Connectivity at temporary work sites is poor and cannot be guaranteed.

O2 The sensor and user device, which are both battery operated, must support continuous operation for several days or even weeks. Frequent charging is not acceptable because of $\mathrm{U} 1$.

O3 The hardware must be extremely robust and be able to operate in adverse conditions (vibrations, heat, water, dust, physical impact). The reason for this of course lies in the fact that the system is used at construction sites in all weather conditions.

\section{SYSTEM DESIGN}

Driven by the set of requirements described in the previous section we designed a system for monitoring HAV using augmented artefacts. From an abstract point of view, our system is a collection of mobile wireless nodes: sensor nodes attached to equipment such as drills, user devices carried or worn by users and mobile gateway units installed in vehicles (see Figure 2). Each device type serves a distinct function:

- sensor nodes measure and interpret vibrations

- user devices record and display data related to a user's vibration exposure

- gateway units store and forward vibration data to the backend infrastructure.

Each node is an autonomous unit capable of operating without any other unit. Yet, if devices come within proximity of each other, they spontaneously establish a communication link. In particular, sensor nodes send vibration data to the user device, and user devices upload user data onto the gateway unit from where it is forwarded to the backend infrastructure for permanent storage.

Figure 3 depicts the system model of the NEMO system. It shows sensor node A within communication range of user devices 1 and 2. Likewise, user device 4 is within communication range of a gateway unit. 


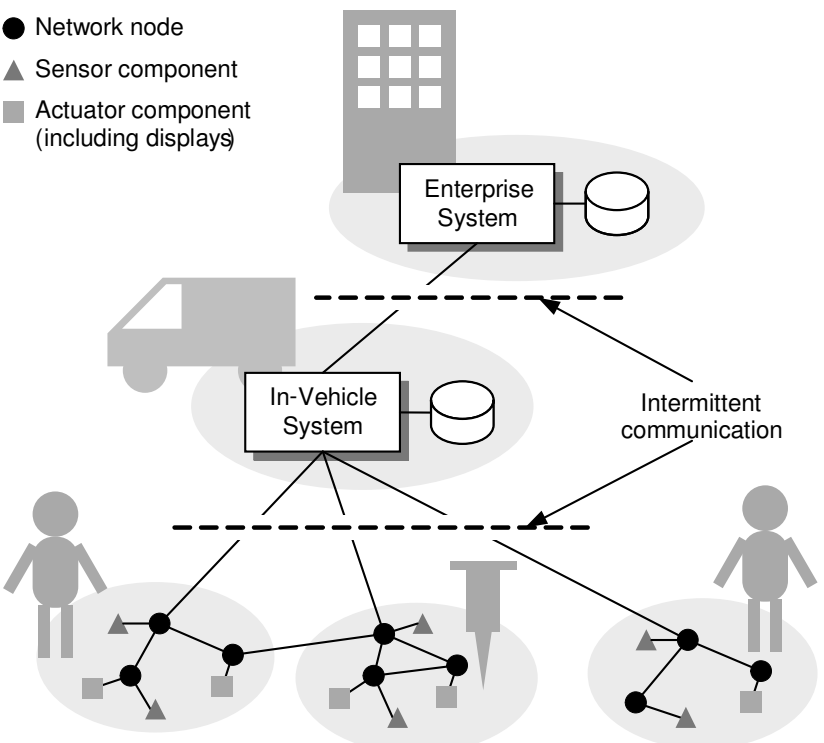

Figure 2: HAV monitoring system

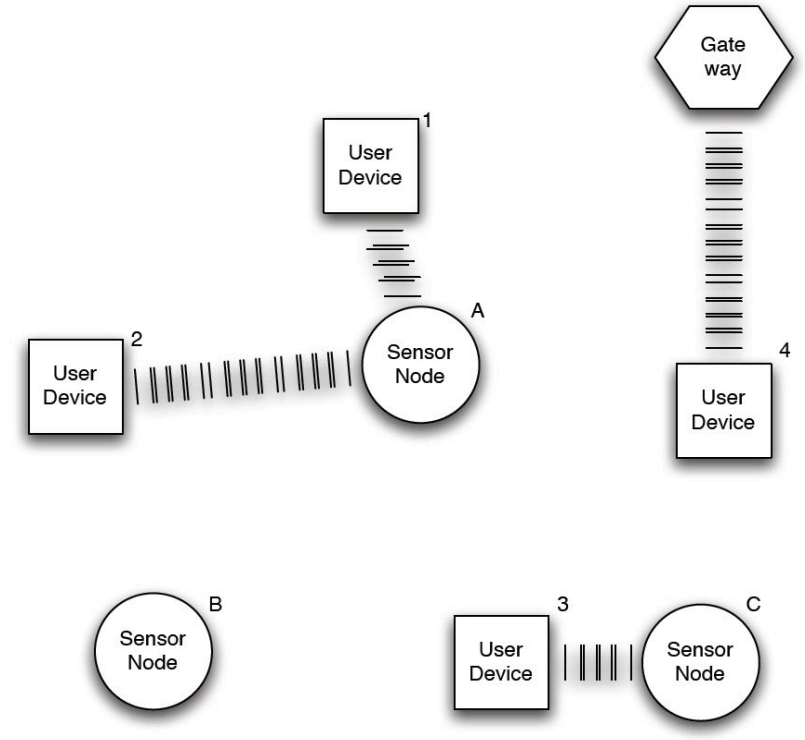

Figure 3: NEMO Devices
A key issue for the NEMO system is to ensure that vibration data is always recorded by the user device of the person who is actually using the equipment. While in practice only one person operates each piece of equipment at each point in time, there may be several 'bystanders', i.e. workers who are in close proximity of the equipment without operating it. In this case, the NEMO system must ensure that data from the sensor node is recorded only on the operators user device and not on the bystanders' devices. We call this the association problem. This problem arises in any systems where it is necessary to determine, for example, which worker is using a specific tool.

We wished to design the system such that the NEMO user device could function as a general purpose H\&S tag. From this perspective, it made little sense to develop a custom solution for solving the association problem between a worker and a drill. For example, the particular characteristics of the drilling scenario, where the tool in operation and the operative experience correlated vibrations, would suggest a solution similar to the one presented in [15] — identifying the tool user, through the correlation of accelerometer readings on both the tool and the operative. Another approach would be to augment the drill handle with a short range RFID reader in order to read an RFID tag from the operative's glove. Although both these approaches would provide a reliable solution to the association problem for this specific application, it would not generalise to other H\&S scenarios.

We decided to implement two distinct approaches to the association problem in order to explore design alternatives. Firstly, we built a solution that required involvement of the drill operator. Specifically, the system required the drill operator to press a button on their personal NEMO device before they started using the drill. The button press was only required when a new worker was about to use the same drill - i.e. on a change of operator. This approach suffers from a number of obvious shortcomings. Most importantly, the requirement for minimal impact on users is not met the workers have to change their work practice due to the new system. In addition, the fact that the association relies on user input makes the system susceptible to human error. However, the approach has the benefit that when used correctly, it provides an unambiguous indication of the tool operator.

The second approach that we implemented was to measure the proximity between the drill and all personal NEMO devices using the wireless interface on each node. This approach was developed on the assumption that over a period of time, the worker operating the drill would spend more time closer to the drill sensor node than all the other workers in the field. This approach appears to satisfy all requirements established by the requirements capture phase, if it can be shown to work reliably in the field.

In addition to the association problem, our abstract design also raises issues with respect to support for "disconnected operation". One of the characteristics of the HAV monitoring systems is that the operating environment is unknown, with minimal or no infrastructural support. For example, a patching gang is normally sent out to perform a task in a company owned vehicle. However, the actual location where the tarmac is to be patched may be quite far from the parked vehicle, depending on traffic conditions and availability of parking spaces. This implies that any infrastructure carried within the vehicle may not always be available to the field components. These characteristics have direct implications on the communication requirements as well as the design of the overall system. Indeed, the mobile nodes that are deployed in the field (i.e. the drill sensor node and the personal NEMO devices) are required to operate without any access to a back-end infrastructure. This implies that the field components should be able to fully realise the H\&S regulations specified by the company, in complete isolation. This indicates a departure from the traditional design of a wireless sensor network where the sensor nodes push their sensor readings to a back-end infrastructure that implements the application logic.

In the developed HAV monitoring system the functionality of monitoring the use of drills in the field, calculating the exposure to $\mathrm{HAV}$, and reporting violations to HAV reg- 
ulations, is fully embedded within the nodes deployed in the field. This approach allows the relocation of the decision making competence from the back-end infrastructure to "where the action is". We have successfully employed this approach in the past to support compliance with H\&S regulations for storage of chemical containers [19]. The benefits of this approach in the context of the HAV monitoring system are:

- responsiveness and immediate decisions when and where they are required.

- low communication requirements and therefore low power requirements.

- simple deployment.

The low range communication that was employed in the development of the field components was sufficient to realise the HAV monitoring system in the field.

\section{IMPLEMENTATION}

\subsection{NEMO Nodes}

\subsubsection{Drill Sensor Node}

The drill sensor node consists of a Particles Smart-it [21], a motes-like wireless sensing node based on a PIC18F6720 microcontroller, with a TR1001 transceiver and an accelerometer sensor. In order to accurately calculate the HAV exposure of a particular user, the drill sensor node is required to

1. identify to user devices the type of drill the sensor is attached to, and

2. measure the exact amount of time the drill is being used.

The former is achieved by assigning a tool ID to the sensor node during installation. The latter requires a mechanism that allows the drill sensor node to identify the exact time that the drill is actually operating (i.e. drilling) and we discuss the design of this mechanism in detail below.

The physical characteristics of the augmented tool played a significant role in the design of the drill's activity recognition mechanism. An obvious approach for identifying when a tool is on or off is to hook into its existing circuitry and monitor when the on/off switch is pressed. However, hydraulic or pneumatic drills do not contain any electrical parts and turning these drills on or off does not involve any electrical circuitry. One possible approach would be to develop our own on/off trigger that would be incorporated in the existing manual on/off switch. However, such an approach would reduce dramatically the deployability of the system. In addition, a common practice for construction companies is to hire tools on demand for performing a particular construction task. This means that any augmentation of such tools should be simple and easy to install; easy enough to be performed by any worker at the time the tools are delivered by the hiring company. For the design of the drill sensor node this meant that it should, ideally, involve a single component that can be easily attached to the tool.

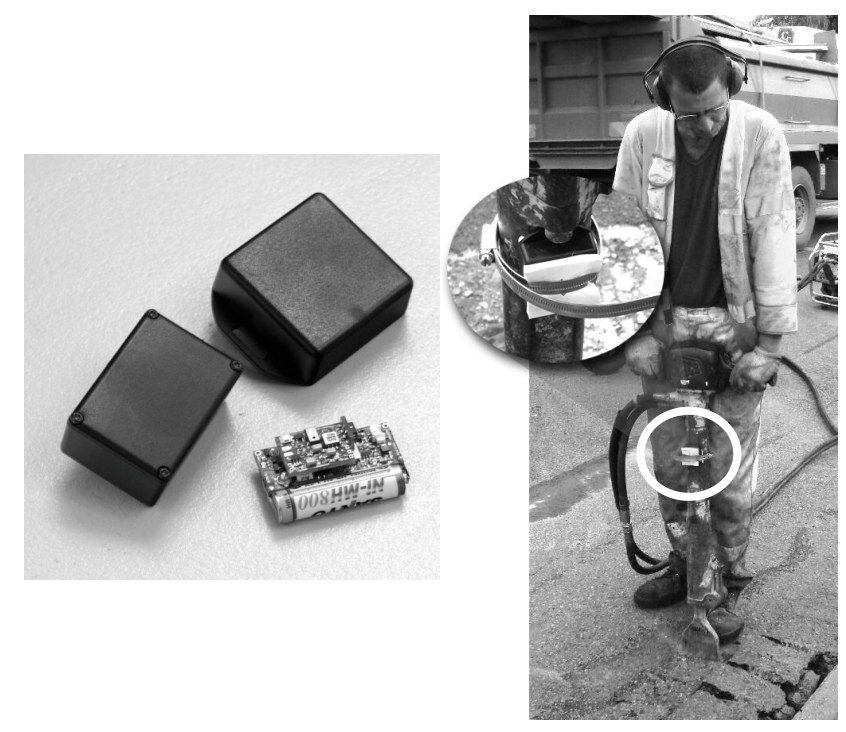

Figure 4: The drill sensor node

With such a requirement in place, the obvious approach was to develop a drill node that would be able to sense the drill's activity based on the tools vibrations. Indeed, the drill sensor node implemented is able to monitor the tool's movements through an accelerometer, and identify the time the drill is actually drilling. When the sensor node discovers that the drill is operating, it measures the time that the tool is in operation. When the drill stops, the sensor node sends the total amount of drilling time to the operative's personal device over the wireless interface.

The drill sensor node is attached to the drill as shown on Figure 4. The node monitors the movement of the drill through the attached accelerometer, capturing acceleration values at a sampling rate of $70-80 \mathrm{~Hz}$. In order to calculate reliably the activity of the drill, the sensor node maintains a sliding window of 50 samples. The identification of the drill's activity involves the calculation of three different metrics:

- Whether the drill is vibrating (true/false): the sensor node checks for periodic up/down movement through the accelerometer values. Specifically, the device measures the number of times the accelerometer values cross the $0 \mathrm{~g}$ point - properly adjusted to account for gravitational acceleration - within the period of the sliding window. If that number is sufficient (more than 5 times) the drill is considered to be vibrating. In order to compensate for any noise on the accelerometer readings, the $0 \mathrm{~g}$ point is defined as values within the range of $(-0.5 \mathrm{~g}, 0.5 \mathrm{~g})$.

- The standard deviation of the accelerometer values: this is used as an indication of the vibration frequency.

- The average amplitude of vibrations.

The drill's state is identified through certain thresholds specified for the three metrics:

$$
\begin{aligned}
\text { drillOn }:= & \text { vibrating }=\text { true } \wedge \text { stdev }>D_{t h} \wedge \\
& \text { amplitude }>A_{t h}
\end{aligned}
$$

where $D_{t h}$ is the threshold for standard deviation and $A_{t h}$ is the threshold for average amplitude. The values of the 


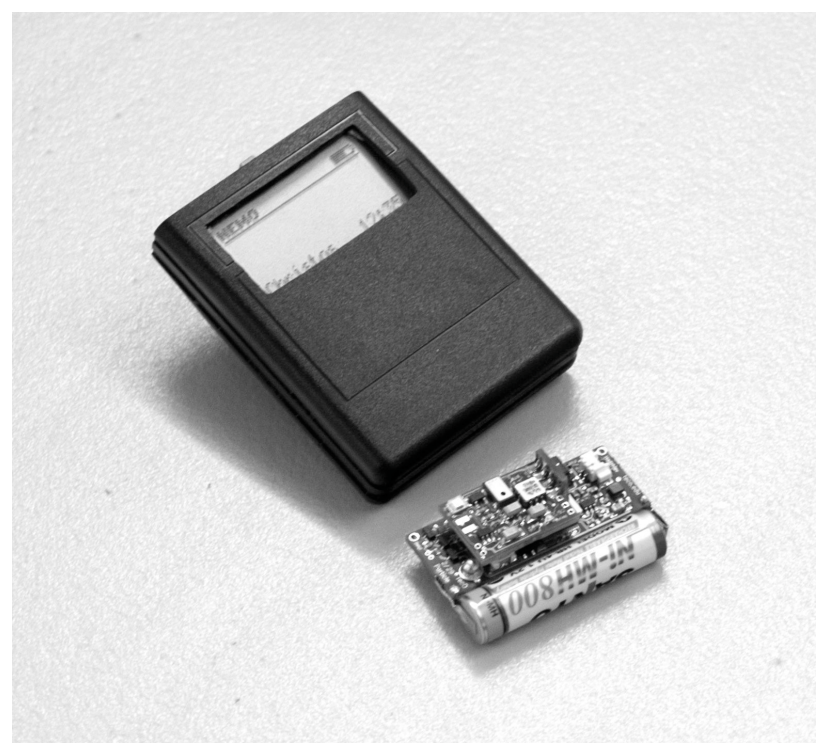

Figure 5: The NEMO user device

thresholds were determined by the system developers during a calibration phase. The specific values that were used are $D_{t h}=7$ and $A_{t h}=3 \mathrm{~g}$. The use of a sliding window of 50 samples and a sampling rate of $70-80 \mathrm{~Hz}$ allows for a timely detection of the drill's state. This in practice means that by the time the drill starts drilling, the sensor node requires approximately less than half a second to detect this ${ }^{1}$.

When drilling starts, the sensor node measures the total drilling time, while at the same time it continues to capture acceleration samples in order to detect the end of drilling. When the drill stops, the sensor node sends a message over the wireless link to the operant's user device with the amount of time that the drill was on.

\subsubsection{User device}

The user device consists of a Particles Smart-it (wireless sensing node), an LCD display, and a button for user interaction. The purpose of the NEMO user device is to collect data about the exposure of the user to vibrations and transfer that data back to the in-vehicle computer (see Figure $5)$. Moreover, it offers an interface to the worker where they can see details about their exposure to vibrations, or alerts in the case where they have violated regulations related to hand-arm vibration.

When the user device is switched on, it can be associated with a particular worker via the in-vehicle computer (or it will fall back to the worker that it was associated with the last time it was used). After this initialisation phase the NEMO device becomes a personalised unit that holds information about the particular worker. In case of a malfunction, the worker can pick a new NEMO device at any time, associate it with himself via the in-vehicle computer and continue working as usual.

In the case when we require manual intervention to form

\footnotetext{
${ }^{1}$ Normally it is not necessary for all values in the window to correspond to the drilling vibrations. In practice when $70 \%$ of the samples in the window (35 samples) correspond to the drilling vibrations the frequency and amplitude values offer a clear indication of the drill's activity.
}

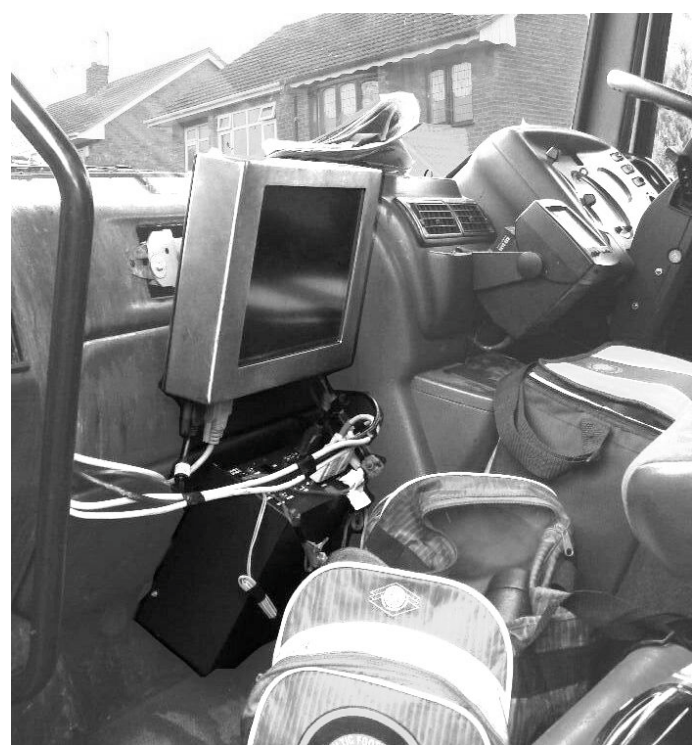

Figure 6: The In-Vehicle computer associations, workers are required to press the button at the top of the device when they take over drilling duties. This button-press switches the NEMO device to vibration monitoring state. In that state the device receives messages from the drill sensor node and records the duration of vibration the worker is exposed to.

The personal NEMO is equipped with $512 \mathrm{~KB}$ of FLASH memory to store the drilling records transmitted by the drill. Each record consists of a timestamp ( 4 bytes), the duration of a drilling burst ( 2 bytes) and the type of the drill (2 bytes). With a record size of 8 bytes, the local memory is capable of holding more than 18 hours of drilling time assuming a worst case scenario of a drilling burst every one second - which is well beyond the duration of an 8 hour shift. At the end of a shift the user device can upload all records related to hand-arm vibration to the in-vehicle unit and through that to the back-end database.

\subsection{In-Vehicle Hub}

We employ an in-vehicle system developed by Lancaster University and In Touch Ltd. The vehicle computer is an Arcom Viper computer [2], running windows CE 5 and utilises a Trakm8 T4 [22] unit installed in the vehicle to communicate with the backend over GPRS (see Figure 6).

The NEMO software that runs on the in-vehicle unit is used to:

1. communicate with the NEMO devices in order to:

(a) Associate user NEMO devices with workers.

(b) Retrieve HAV exposure records from the NEMO devices at the end of their shift.

2. communicate with the back-end database in order to upload HAV exposure records.

In general, the workers are required to use the invehicle application only at the beginning and end of their shift. At the beginning of their shift, they need to use the application in order to select the worker that is associated with each 


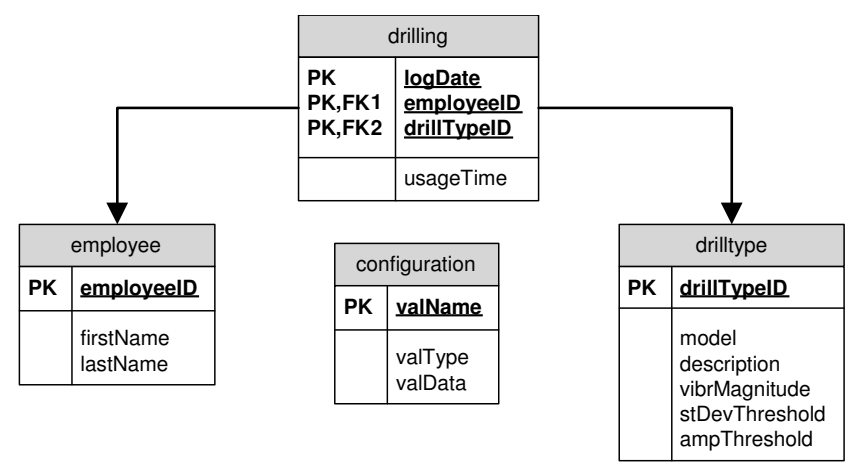

Figure 7: The back-end database

NEMO device. At the end of their shift, they select the uploading function that retrieves all HAV records from the NEMO devices and transmits them to the back-end over the T4 unit.

\subsection{Back-End Database}

The HAV monitoring system provides a feed that can be used to produce management systems. One of the NEMO project partners (in conjunction with the University) has developed a combined database and web access system that stores all the information collected by the remote NEMO objects and allows operators at central offices to view and use this data.

As discussed earlier, at the end of each shift the personal NEMO units worn by the operators upload information regarding the worker's exposure to vibrations to the in vehicle unit. This unit then communicates this information via GPRS to a back-end server which stores it in a database. The drilling events are registered in the database as records that include the ID values for the tool and operator, time stamped together with the usage time (Figure 7).

Access to the information stored in the database is provided via a web front end that allows authenticated users to modify or add new information about operators and drills, as well as inserting configuration data that can be transmitted to the remote units. The users can also query the database to produce usage logs for tools and operators, as well as periodical and exception reports that can assist managers in identifying problem areas.

\section{EVALUATION}

The evaluation of the HAV monitoring system involved a small scale deployment of the system in a road maintenance patching gang. The field trial was used as both an evaluation of the prototype HAV system and to gain an understanding of the use of this technology in the field under real conditions. The following sections present our evaluation methodology and our results.

\subsection{Methodology}

The field trial deployment was organized in the following phases:

\section{Phase I-Calibration.}

The calibration phase of the field trial was conducted on the 10 August 2006. The main aims of the calibration were to collect vibration recordings from the hydraulic drill that was used during the field trial and to experiment with the appropriate technique for mounting the sensor node on the drill. The vibration recordings collected at this phase were used for the proper configuration of the drill sensor node.

During the calibration, a drill was augmented with a sensor node that could capture acceleration values and transmit them to a nearby laptop over a wireless link (Figure 8). The drill sensor node was mounted on the main body of the drill, using a jubilee clip (Figure 4). A drill operator was asked to use the drill to break different surfaces (concrete, tarmac).

\section{Phase II - Pre-Trial.}

The pre-trial was conducted on the 15-16 August 2006. The main purpose of the pre-trial was to test the operation of the system before the actual field trial. During the pretrial, we:

- Tested the operation of the drill sensor node and evaluated its accuracy in detecting when the drill is on or off.

- Tested the drill - user NEMO association mechanism to see if it functions as expected in the field.

- Tested the communication between the NEMO devices and the in-vehicle computer.

During the pre-trial, the system was not handed to the workers at all. The method that we used was to have members of our team shadowing the workers and handling the NEMO user devices ourselves. This allowed us to observe the system's operation and identify any unexpected behaviour.

\section{Phase III - Field Trial A.}

The first phase of the field trial was conducted for one week on the 11-15 of September 2006 (see Figure 9). During the first phase of the field trial, all system components were in place. Specifically, the in-vehicle computer and the T4 unit were installed in the patching gang's van. During the field trial, we allowed the workers to operate the system themselves without our involvement. The main aim of the first phase of the field trial was to conduct a thorough evaluation of the system's behaviour. For that purpose, we used

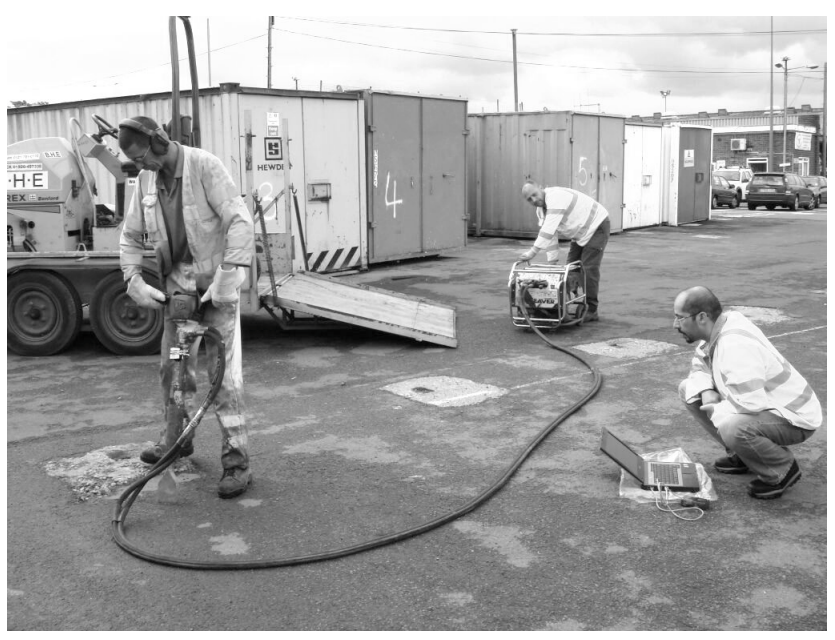

Figure 8: Calibration 


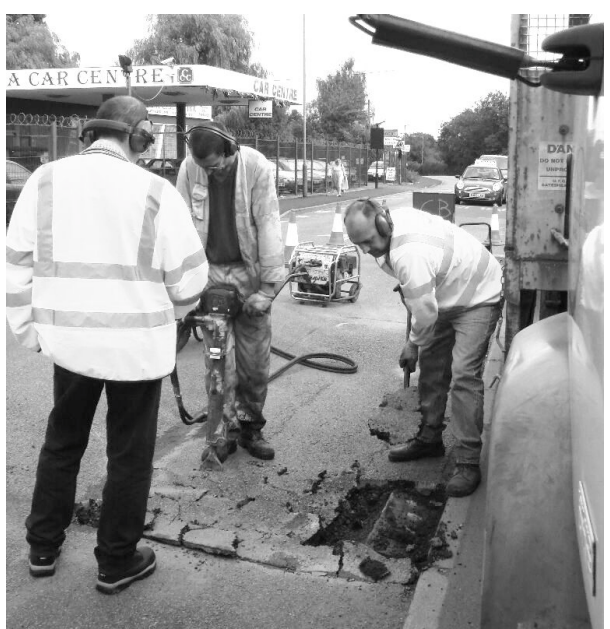

Figure 9: Field Trial

a video camera that recorded all field activities. We were then able to compare the data collected from the NEMO system against the video footage, and check the correctness of the recordings.

\section{Phase IV - Field Trial B.}

The second phase of the field trial was conducted for one week on the 25-28 September 2006. The main purpose of the second phase of the field trial was to experiment with alternative techniques for addressing the association problem. Specifically, during the second phase of the field trial, we abandoned the association mechanism that required the worker to press a button before using the drill. The experiment conducted had as a main aim to identify the worker that is closer to the drill during the time that the drill is operating. The assumption was that the proximity could be established in relation to the quality of the communication link between the drill sensor node and the personal NEMO devices of the workers in the field. Based on that, we setup an experiment where we measured the number of lost packets for all communications between the drill and the NEMO devices and compared the results against the ground truth from the video footage.

\subsection{Results}

\subsubsection{Communication}

Even before deploying the system in the field, we were aware that the communication of the mobile nodes would be greatly affected by the conditions in the field. Specifically, we were aware that attaching the drill sensor node on a hydraulic drill, a heavy metallic object, would influence the $\mathrm{RF}$ communication. Indeed, during the calibration phase and the pre-trial we identified that the communication range between the drill sensor node and the personal NEMOs was significantly shorter than the range these units had in the lab. While the communication range between the nodes could reach a distance of more than $20 \mathrm{~m}$ in the lab, the effective distance in the field was less than $5 \mathrm{~m}$.

One of the results that we gathered from the calibration phase was that the communication range of the drill sensor node was significantly shorter when the drill was operating.
In particular, communication with the drill sensor node was at the range of 4-5 $\mathrm{m}$ when the drill was idle, and less than $2 \mathrm{~m}$ when the drill was vibrating. Although we were aware that the crystal oscillator used by an RF transceiver is sensitive to physical vibrations [9], this calibration phase gave us a clear indication of the extent to which the RF communication was affected by vibrations.

This observation affected the design of the communication protocol that was employed between the field nodes during the field trial. In order to overcome the degradation of the communication link during drilling, the communication protocol between the drill sensor node and the personal NEMO required the exchange of messages only at the end of a drilling burst (when the drill is not vibrating). Moreover, in order to compensate for the low communication quality and high loss of packets, we decided to increase redundancy on the transmitted data. Indeed, the protocol for transmitting vibration data from the drill to the personal device, involved multiple transmissions of each packet (each packet sent three times). Moreover, each packet contained the total amount of time the particular worker has been using the drill on that day. This approach reduced the significance a single lost packet would have on the recorded data: any data contained in a lost packet could be compensated by the next received packet.

\subsubsection{Robustness and Reliability}

The environment where the HAV system was deployed is a harsh environment. The tools in particular, are exposed to rough treatment and bad weather conditions. Augmenting the drill that would be exposed to such conditions was certainly an issue that had to be considered. In order to protect the drill sensor node we used ABS casing, IP-54 certified (protection against dust and water), with $3 \mathrm{~mm}$ thickness. The field trial provided anecdotal evidence that the choice we made for the casing was sufficient for enduring the harsh conditions in the field - we used the same sensor node for the whole two-week period of the field trial and at the end of the field trial the sensor node showed no signs of damage.

The requirement for robustness was less important for the user NEMO devices. The workers had the tendency to treat them like they would with their mobile phones: placed safely in their jacket's pocket away from any immediate harm. However, in order to limit the effect that a destroyed user devices would have in the field, we decided to build them as "easily replaceable" commodities. In the deployed system the workers were able to replace any malfunctioning user device at any time with a new one available in their vehicle. The new device could be personalised using the in-vehicle computer, allowing the worker to continue their work as before. A malfunction in one of the personal NEMOs during the pre-trial allowed us to test the replaceability of the personal NEMOs. In that incident, we were able to bring a new personal NEMO in the field, personalise it through the in-vehicle computer and continue the field trial normally.

\subsubsection{Power consumption}

During the two week field trial, the units deployed could maintain a battery lifetime of approximately one day (28 hours for the drill unit and 17 hours for the user device). This lifetime was considered sufficient, on the assumption that all field nodes could be recharged at the end of the shift. However, our observations during the field trial re- 
vealed that there was a clear asymmetry between the different types of devices in terms of battery lifetime requirements. Specifically, the personal NEMO devices were much easier to recharge, as the workers could easily leave them in a recharger over night. In contrast, recharging the drill device included the cumbersome task of dismounting and remounting the device on the drill. This fact gave us a clear indication that in order to reduce the overhead this system had on the workers, it would be imperative to maintain a much longer battery life for the drill device.

The functional characteristics of the drill device offers the opportunity to dramatically improve the battery lifetime of the device. Specifically:

- The communication requirements for the drill device are minimal. The communication interface is only required to be on for fractions of seconds at the end of every drilling burst. The rest of the time the wireless communication can be switched off while the microcontroller detects the drill's activity to check whether the drill is being used.

- The vibrations of the drill can be used as a source of power. Appropriate hardware can be used to harvest the energy produced during drilling to recharge the drill's battery.

We intend to improve the battery lifetime of the drill unit by the design of bespoke hardware that will take advantage of the mentioned characteristics. Specifically, shutting down the RF module on the drill node can improve significantly the battery lifetime of the device. Preliminary results show that by employing this technique it is possible to achieve a battery life of more than 6 months.

\subsubsection{Accuracy}

Evaluating the accuracy of the HAV system involved the comparison of the data we got from the system against the ground truth. During the field trial, we established a clear indication of the ground truth through a video footage of

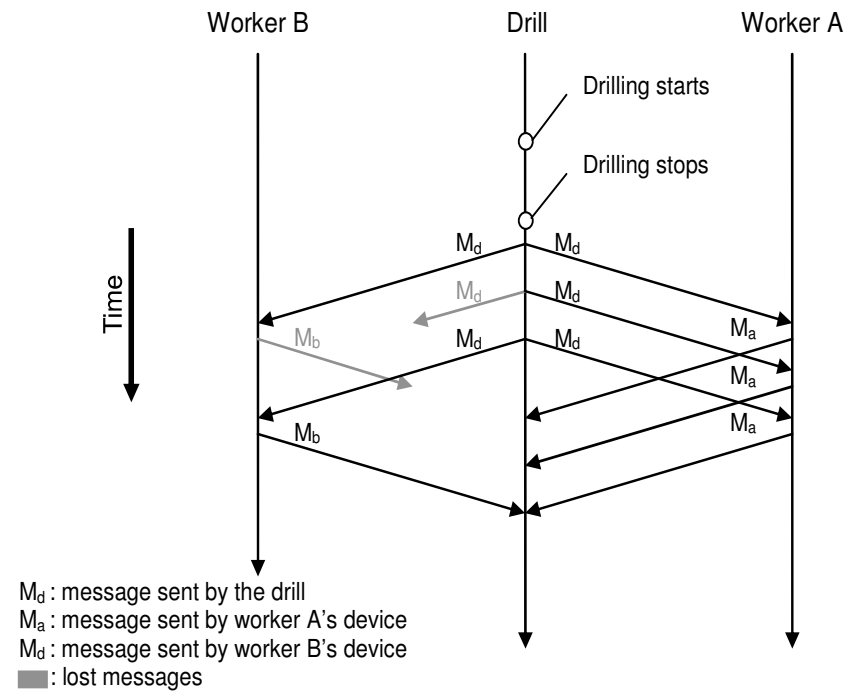

Figure 10: Communication protocol for the proximity detection experiment the activities in the field. We were then able to compare the readings from the system against the video footage.

During the post-trial analysis, we edited the video footage cutting out the periods of time that the drill was not active. Combining the video clips of individual drilling bursts, we got a single video that showed us the total drilling time required to work on a single tarmac patch. We were then able to compare this time against the total time recorded by the system for the particular tarmac patch.

Through the post-trial analysis, we were able to establish that the drilling time recorded by the HAV system had an average error of $3.2 \%$ against the actual drilling as recorded by the video footage. Furthermore we established that the error was proportional to the total number of drilling bursts involved in the patching of a single patch, and inversely proportional to the duration of the drilling bursts. This meant that working on a tarmac patch with a small number of drilling bursts of longer duration would result on a smaller error than a large number of drilling bursts of shorter duration. Based on these observations, we believe that the error is a constant value for each drilling burst and we have been able to modify the drilling time measurement in order to add a compensation for the measurement error.

\subsubsection{Proximity Detection}

During the second week of the field trial, we conducted an experiment trying to infer the operator of the drill based on their proximity to the drill sensor node. The approach used was based on the quality of the communication between the drill sensor node and the user NEMO devices of the workers in the field. The quality of the communication is estimated according to the number of lost packets on each communication link. The proximity detection is based on the assumption that the communication quality is relative to the distance between two wireless nodes.

\section{Setup of the Experiment.}

In this experiment both the drill sensor node and the user devices were modified in order to record the number of mes-

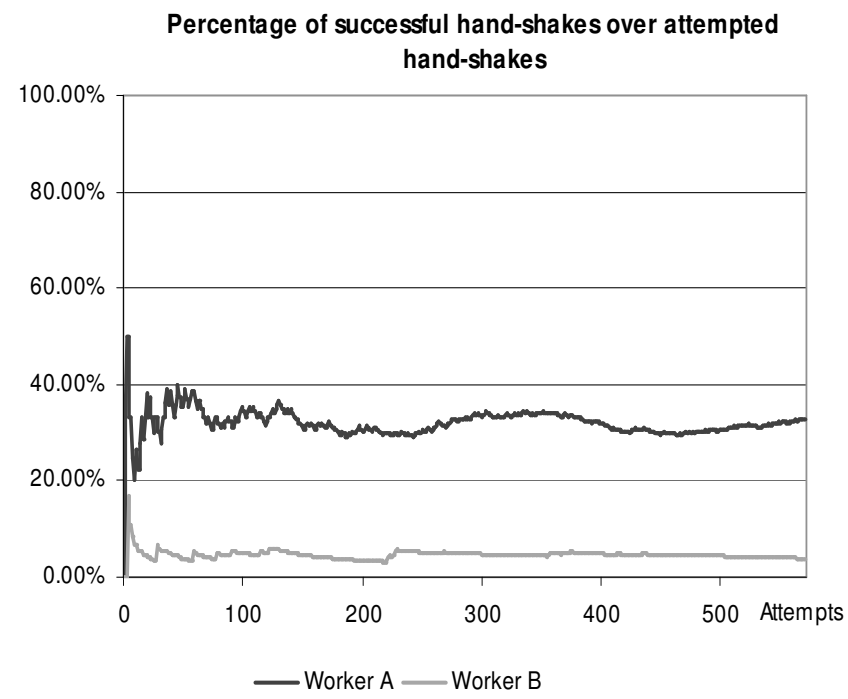

Figure 11: Percentage of successful hand-shakes over the period of one tarmac patch 

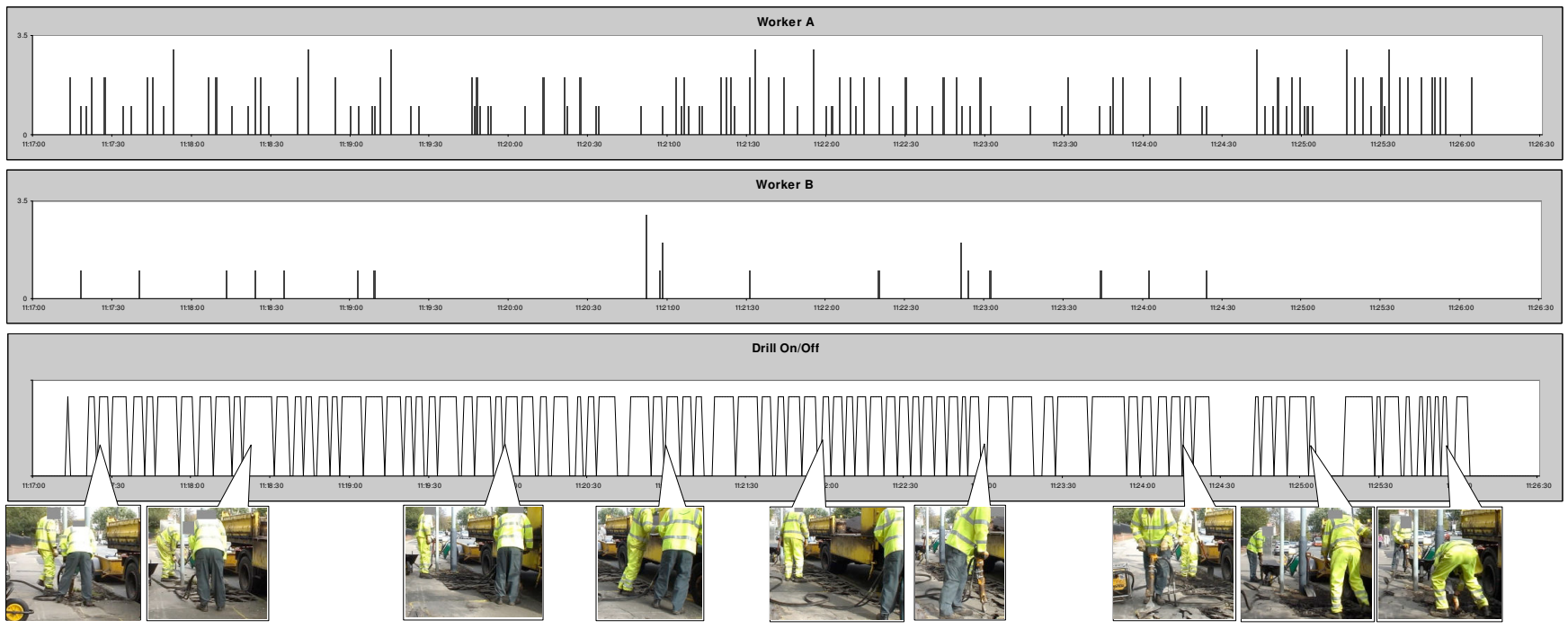

Figure 12: Comparing the number of received packets against the video footage. The blue lines represent the received packets from the worker operating the drill while the white lines represent the packets from the other worker. The lower graph shows the drill's activity (on/off).

sages exchanged between the nodes during operation. Moreover, a protocol was implemented that allowed the sporadic "hand-shake" between the drill sensor node and all close-by user NEMO devices.

During the calibration phase of the field trial, we established a significant difference in communication quality between the drill's idle and operating time. In order to get a valid result from this experiment, we refined the protocol to avoid any communication during drilling time. Specifically, when the drill is operating the sensor node monitors the drill's activity and awaits the time that the drill stops. When the drill stops the sensor node broadcasts a message with the total drilling time (repeated 3 times). On receipt of that message, all user devices reply back with an acknowledgement (Figure 13). The drill sensor node records the number of replies it received from each user NEMO device. The aim of the experiment is to record the number of successful "hand-shakes" between the drill and the user devices, over the period of drilling one tarmac patch. The assumption is that the worker using the drill does not change until the whole patch is finished. By analysing the recorded data, we want to check the feasibility of this approach as a possible technique for proximity detection

\section{Results}

The experiment was conducted for four consecutive days during Phase IV. During this time, there were two workers in the field equipped with a user NEMO device. The data that we collected included time stamped records of the successful handshakes exchanged between the drill and the two workers in the field. During this time, we used a video camera that recorded all activities in the field. We were then able to compare our measurements with what was happening in the field.

The first step in the post-trial analysis was to compare the number of successful handshakes from the two workers. As shown in Figure 11, there was a clear difference between the worker operating the drill (worker A) and the other worker (worker B). Specifically, in the results shown in the figure, upon completion of a tarmac patch, worker A had in average $32.8 \%$ successful hand-shakes against $3.9 \%$ for worker B. In general, for all the tarmac patches for which we ran the experiment the worker operating the drill had a rate of successful handshakes varying in the range of $26 \%-40 \%$ while the rate for the other worker varied in the range of $0 \%$ $-5 \%$.

The next step in the analysis was to align the timestamped records of successful handshakes against the video footage (Figure 12). Through this, it became clear that there was a direct relation between the quality of communication and the workers proximity to the drill, as well as their orientation towards the drill. Indeed, the number of lost packets between the drill and the personal NEMOs increased significantly when the workers were more than $2 \mathrm{~m}$ further from the drill. Similarly, when the workers were not facing the drill and therefore their body was obstructing the communication between their personal NEMO and the drill sensor node, the communication deteriorated.

These results showed that the number of lost packets is a valid indicator for identifying the drill operator. Throughout the four days of experiments, it would have been possible to identify successfully the person operating the drill using as an indication the number of successful handshakes exchanged with the drill sensor node. Based on these results, a very simple algorithm for establishing association using proximity, would involve the aggregation of the number of successful handshakes over the period of drilling a single patch. This way the worker with more successful handshakes would be identified as the operator of the drill.

\subsubsection{Usability Issues}

The second week of the field trial gave us the opportunity to get a direct feed-back on the form factor of the personal NEMO device. To provide a point of comparison, we replaced the personal NEMO device with a wrist mounted device for one day (Figure 13). This allowed us to compare 


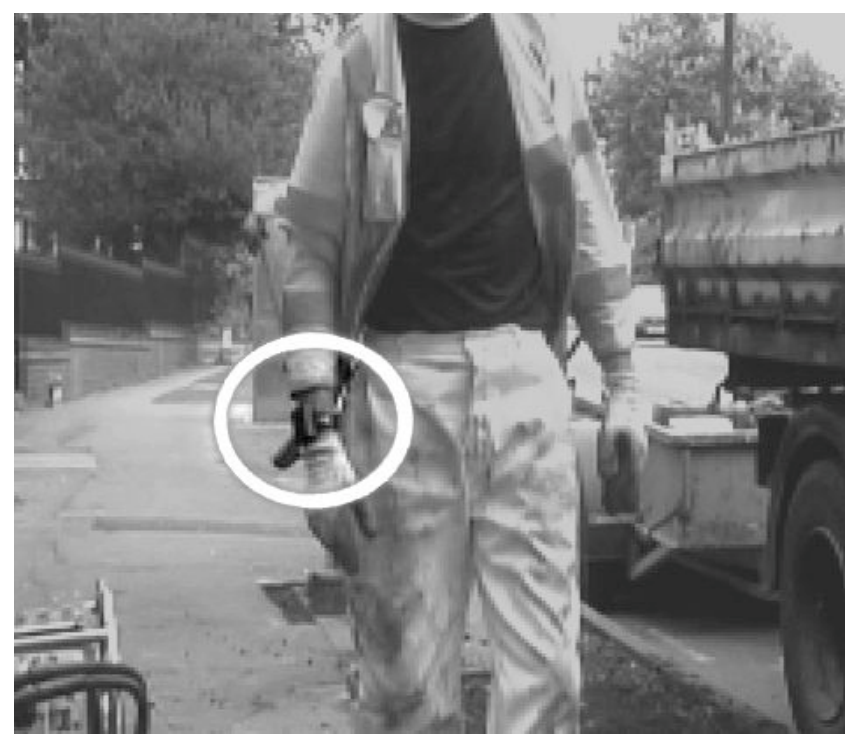

Figure 13: A worker wearing a wrist mounted NEMO device

the workers' response to the two different unit types. The workers showed a strong preference for the original NEMO device. They found it non intrusive as they could easily put it in their pocket. Moreover, the LCD display allowed them to get immediate feedback on the levels of their HAV exposure.

When the workers were asked to use a wrist mounted unit, they showed an immediate negative attitude towards the device. They complained that having something on their wrist felt more like a surveillance device than a H\&S support system. Their general comment was that the wrist mounted device "felt awkward" and it gave them a feeling that they are being monitored on whatever they do. This attitude did not come as a surprise to us. Through conversations with the workers we gathered indications that an automated $\mathrm{H} \& \mathrm{~S}$ monitoring system is perceived by the workers as a special purpose surveillance system. The wrist mounted unit emphasised this perception as it increased their sense of being "tagged".

Our observations during the field trial gave us an indication of significant factors that can affect the acceptability of the end system. Such factors are:

- Familiarity of the form factor: the original user device had a form factor similar to a common mobile phone. This allowed the workers to relate better with the particular device.

- Lack of restrictions: the wrist mounted unit required to be worn on a particular way. On the contrary, the original user device could be placed anywhere they found convenient.

- Display: in order to maintain a smaller footprint, the wrist mounted unit did not have a display. This limits the workers' awareness of the functionality of the device. Moreover, it raised fears that it was doing more than just recording HAV exposure.

These results justified our initial approach of not designing a system that required any hand mounted devices but rather follow a design where personal devices can be handled in a more liberal manner. It is our belief that this approach can significantly improve the acceptability of the HAV system.

\section{RELATED WORK}

Ubiquitous computing in industrial workspaces has been mostly related to asset management. For example, industrial artefacts have been augmented at very low cost with RFID tags [14] to support their unique identification and tracking in an appropriately instrumented environment. Our approach however, is to augment artefacts with sensing, computing and networking capabilities, allowing applications that are fully embedded within the artefacts with minimal infrastructural support. This approach has been employed in the past for monitoring storage regulations in a chemical plant [19]. In the work presented in this paper, we employ this approach in the context of work activities, involving wearable user devices along side augmented physical objects. This approach introduces new challenges (e.g. the association problem, usability issues) and new opportunities to resolve such issues through cooperating mobile nodes.

Our general approach is related to other ubiquitous computing research concerned with instrumentation of the world. Most of these systems and infrastructures are based on instrumentations of controlled environments such as offices [1, 4] or homes [20].

Previous research has also considered the role of artefacts in connection to location and users. The Cooltown project [12] investigates the digital presence of people, places and things. The SPECs project [13] proposes a platform for activity sensing using peer-to-peer proximity. More closely related to our work are systems directly concerned with artefacts and their situation, for example tracking of movable assets and innovative business services [8, 14].

In the domain of HAV, there are many commercially available equipment for measuring Hand-Arm vibrations [7, 3, 18]. These are highly accurate, but rather bulky and expensive devices, not targeted for continuous every day use. Their purpose is to allow field engineers to test their tools and measure their vibration magnitude. These measurements are then used in order for the company to specify regulations about the time these tools should be operated. The HAV monitoring system presented in this paper, serves a different purpose. The aim of this system is to monitor the compliance with the regulations specified on a daily basis with minimum effort or obstruction from the workers.

There is related ubiquitous computing research concerned with assessment of critical situations, such as fire fighting $[11,6]$, avalanche rescue $[17]$ and guidance through dangerous terrain [16].

\section{CONCLUSIONS}

In this paper we have described our experiences of designing and deploying the NEMO Hand Arm Vibration (HAV) monitoring system. The system offers an integrated architecture for capturing HAV exposure data in the field, providing feedback about exposure levels both in the field and as input to a back-end database, and the system has been the subject of a successful two week field trial. Our major result is that we have been able to show that a functioning HAV H\&S compliance system can be constructed using an approach based on augmented artefacts. We believe this ap- 
proach has significant potential for other H\&S domains and our future aim is to evaluate the reusability of the NEMO nodes as building blocks for the development of new H\&S applications. In addition to this major result, we have also been able to report on a wide range of experiences we gained during this design and deployment exercise, which we believe will be of value to researchers planning to work in the area of mobile $\mathrm{H} \& \mathrm{~S}$ systems.

\section{ACKNOWLEDGEMENTS}

This work has been supported by the UK Engineering and Physical Science Research Council (EPSRC) project NEMO (EP/C014677/1) and the EU project CoBIs (IST 004270). We would like to thank all the partners in the NEMO and CoBIs projects.

\section{REFERENCES}

[1] M. Addlessee, R. Curwen, S. Hodges, J. Newman, P. Steggles, A. Ward, and A. Hopper. Implementing a sentient computing system. IEEE Computer, 34(8):50-56, Aug. 2001.

[2] Arcom. Viper. http://www.arcom.com/ pc104-xscale-viper.htm [sited: 04/12/2006].

[3] Brüel and Kjær. Hand-Arm Vibration Meter - type 2537. http://www.bksv.com/pdf/Bp1695.pdf [sited: 04/12/2006].

[4] J. R. Cooperstock, S. S. Fels, W. Buxton, and K. C. Smith. Reactive environments: Throwing away your keyboard and mouse. Communications of the ACM, 40(9):65-73, Sept. 1997.

[5] N. Davies, C. Efstratiou, J. Finney, R. Hooper, G. Kortuem, and M. Lowton. Sensing danger challenges in supporting health and safety compliance in the field. In Proceedings of the $8^{\text {th }}$ IEEE Workshop on Mobile Computing Systems and Applications (HotMobile 2007), Tucson, Arizona, Feb. 2007.

[6] D. Doolin and N. Sitar. Wireless sensors for wildfire monitoring. In Proceedings of SPIE Symposium on Smart Structures and Materials, San Diego, CA, Mar. 2005.

[7] EPM. VIS-015 hand arm vibration meter. http://www.epm-uk.com/enviro/vis015/handarm.htm [sited: 04/12/2006].

[8] A. Fano and A. Gershman. The future of business services in the age of ubiquitous computing. Communications of the ACM, 45(12):83-87, Dec. 2002.

[9] R. L. Filler. The acceleration sensitivity of quartz crystal oscillators: A review. IEEE Transactions on Ultrasonics, Ferroelectrics, and Frequency Control, 35(3):297-305, May 1988.

[10] HSE. Hand-arm vibration: Control of Vibration at Work Regulations 2005. Guidance on Regulations L140. Statutory Instrument 2005 No. 1093. Health and Safety Executive, 2005.

[11] X. Jiang, N. Y. Chen, K. Wang, L. Takayama, and J. A. Landay. Siren: Context-aware computing for firefighing. In Proceedings of Second International
Conference on Pervasive Computing (Pervasive 2004), volume 3001 of Lecture Notes in Computer Science, pages 87-105, Vienna, Austria, Apr. 2004.

[12] T. Kindberg, J. Barton, J. Morgan, G. Becker, D. Caswell, P. Debaty, G. Gopal, M. Frid, V. Krishnan, H. Morris, J. Schettino, B. Serra, and M. Spasojevic. People, places, things: Web presence for the real world. Mobile Networks \& Applications (MONET), 7(5):365-376, Oct. 2002.

[13] M. Lamming and D. Bohm. SPECx: Another approach to human context and activity sensing research. In Proceedings of the $5^{\text {th }}$ International Conference on Ubiquitous Computing (Ubicomp 2003), volume 2864 of Lecture Notes in Computer Science, pages 192-199, Seattle, WA, USA, Oct. 2003. Springer.

[14] M. Lampe and M. Strassner. The potential of rfid for movable asset management. In Proceedings of Workshop on Ubiquitous Commerce at Ubicomp 2003, pages 9-12, Seattle, Oct. 2003.

[15] J. Lester, B. Hannaford, and G. Borriello. "Are you with me?" - Using accelerometers to determine if two devices are carried by the same person. In Proceedings of Second International Conference on Pervasive Computing (Pervasive 2004), volume 3001 of Lecture Notes in Computer Science, pages 33-50, Vienna, Austria, Apr. 2004. Springer Berlin.

[16] Q. Li, M. DeRosa, and D. Rus. Distributed algorithms for guiding navigation across a sensor network. In Proceedings of the Ninth Annual International Conference on Mobile Computing and Networking, (MOBICOM 2003), pages 313-325, San Diego, CA, USA, Sept. 2003. ACM.

[17] F. Michahelles, P. Matter, A. Schmidt, and B. Schiele. Aplying wearable sensors to avalanche rescue: First experiences with a novel avalanche beacon. Computers E Graphics, 27(6):839-847, Dec. 2003.

[18] Quest Technologies. HAVPro personal human vibration monitor. http://www.questtechnologies.com/Vibration/hav_pro.htm [sited: 04/12/2006].

[19] M. Strohbach, G. Kortuem, H. W. Gellersen, and C. Kray. Cooperative artefacts: Assessing real world situations with embedded technology. In Proceedings of the $6^{\text {th }}$ International Conference on Ubiquitous Computing (Ubicomp 2004), volume 3205 of Lecture Notes in Computer Science, pages 250-267, Nottingham, U.K., Sept. 2004.

[20] E. M. Tapia, S. Intille, and K. Larson. Activity recognition in the home using simple and ubiquitous sensors. In Proceedings of Second International Conference on Pervasive Computing (Pervasive 2004), volume 3001 of Lecture Notes in Computer Science, pages 158-175, Vienna, Austria, Apr. 2004.

[21] Teco. Smart-its particle. http://particle.teco.edu/ [sited: 04/12/2006].

[22] Trackm8. T4. http://trakm8.com/s.nl/sc.8/ category.50/.f [sited: 04/12/2006]. 